\title{
Curación de noticias en el correo electrónico: análisis de newsletters periodísticas españolas
}

\author{
News curation by email: Analysis of the Spanish journalistic \\ newsletters
}

Javier Guallar. Universidad de Barcelona. España

jguallar@ub.es

$[\mathrm{CV}]\left(\mathrm{G} \mathrm{R}^{\mathrm{G}} \mathrm{O}\right.$

Laura Anton. Betevé. España.

lanton@beteve.cat

[CV] $\mathrm{G}$

Rafael Pedraza-Jiménez. Universidad Pompeu Fabra. España

rafael.pedraza@upf.edu

[CV] $\mathrm{C}$

Mario Pérez-Montoro. Universidad de Barcelona. España

perez-montoro@ub.edu

$[\mathrm{CV}]$ (1) G $\mathrm{B}_{\mathrm{a}}$

Este estudio forma parte del proyecto "Narración interactiva y visibilidad digital en el documental interactivo y periodismo estructurado" RTI2018-095714-B-C21 (MICINN/FEDER), España (2019-2021); y cuenta con el apoyo del grupo de investigación consolidado "Cultura i Continguts Digitals" (SGR 2017-422), financiado por la Agència de Gestió d'Ajuts Universitaris i de Recerca (AGAUR) de la Generalitat de Catalunya.

Cómo citar este artículo / Referencia normalizada

Guallar, J., Antón, L., Pedraza-Jiménez, R. y Pérez-Montoro, M. (2021). Curación de noticias en el correo electrónico: análisis de newsletters periodísticas españolas. Revista Latina de Comunicación Social, 79, 47-64. https://www.doi.org/10.4185/RLCS-2020-1488

\section{RESUMEN}

Introducción: Las newsletters o boletines electrónicos son un producto de creciente importancia en la actualidad en los medios de comunicación digitales y constituyen un canal muy propicio para la realización de la curación de contenidos, uno de los servicios considerados más relevantes en el periodismo digital del siglo XXI. La finalidad de este trabajo es analizar cuál es el uso de la curación de contenidos periodística por parte de las newsletters de la prensa española. Metodología: Para ello se ha realizado una investigación de metodología evaluativa, que se basa en un sistema de análisis compuesto de parámetros e indicadores organizados en torno a dos grandes áreas o dimensiones: Contenido y Curación. Se han analizado durante dos meses todas las newsletters (84) de 16 diarios españoles de relevancia, tanto tradicionales como nativos digitales. Resultados: Los resultados muestran una radiografía de las características de la curación periodística en cuanto a la cantidad de contenidos curados, el rango temporal de los mismos, la procedencia y las fuentes de información 
empleadas, la autoría, las técnicas de curación utilizadas y el uso informativo de los enlaces. Discusión y conclusiones: Entre las conclusiones, se presenta cómo es el boletín periodístico tipo, se ofrecen clasificaciones de los mejores boletines y de medios y se avanza en la conceptualización de la curación, concluyendo con la existencia de dos tipos de curación: intelectual y automatizada.

PALABRAS CLAVE: periodismo digital; medios de comunicación; prensa digital; diarios; curación de contenidos; boletines; fuentes de información.

\begin{abstract}
Introduction: Newsletters or electronic bulletins are currently a product of growing importance in digital media and represent a channel that is highly conducive to the curation of content, which is one of the services that is considered to be most relevant in 21 st century digital journalism. The main goal of this work is to analyze the use of journalistic content curation in newsletters emitted by the Spanish press. Methodology: To achieve this goal, a study using an evaluative methodology has been carried out, based on an analysis system composed of parameters and indicators organized along two major areas or dimensions: Content and Curation. All the newsletters (84) from 16 relevant Spanish newspapers, both traditional and native digital, have been analyzed for two months. Results: The results provide a snapshot of the characteristics of journalistic curation in terms of the amount of curated content, its time range and origin, the information sources, the authorship, the curation techniques, and how links are used to provide information. Discussion and conclusions: Based on the results of this analysis, we are able to depict the typical journalistic bulletin, offer a ranking of the best Spanish newsletters and the media, and propose a conceptualization of content curation, revealing the existence of two types of curation: intellectual and automated.
\end{abstract}

KEYWORDS: digital journalism, media, digital press, newspapers, content curation, newsletters, information sources.

\title{
CONTENIDO
}

1. Introducción. 2. Objetivos. 3. Metodología. 4. Resultados. 5. Discusión y conclusiones. 6. Referencias

\section{Introducción}

Las newsletters o boletines electrónicos son un producto veterano en el periodismo digital. En su momento "fueron uno de los primeros formatos especiales que los periódicos digitales afrontaron cuando hace más de dos décadas dieron el salto al entorno web" (Trillo-Domínguez y AlberichPascual, 2020), si bien pasaron después a un segundo plano y es en los últimos años cuando están viviendo un auténtico resurgimiento (Jack, 2016), en una situación que algunos analistas han calificado de "madurez de los boletines como medio de comunicación" (Pellicer, 2018).

Este interés actual de las empresas de medios por las newsletters se explicaría por algunas características propias que las diferencian de otros productos periodísticos. Según datos recogidos en los recientes Digital News Reports de 2019 y 2020 (Newman et al. 2019; Newman et al. 2020), una tercera parte de las audiencias consulta las noticias en el propio medio (web o app) y las otras dos terceras partes mediante plataformas sociales, buscadores o el correo electrónico, siendo este último canal al que llegan, sin competencia, las newsletters, en una tendencia que refleja la importancia creciente que está adquiriendo el correo electrónico en la vida diaria (Newman, 2020). Recientes investigaciones corroboran que los lectores "aprecian las ediciones finitas" (Súarez, 2020): frente a otros canales, desde el sitio web a los medios sociales, en los que la información se presenta 
fragmentada y cambiante, la newsletter pone "orden en el caos" (Carr, 2014) y muestra la información de una manera "finita, jerarquizada, generalmente breve, directa" (Laboratorio de Periodismo, 2019). A ello hay que sumar que se trata de una comunicación personalizada e intencional, ya que han sido los propios lectores quienes han pedido específicamente recibir en sus correos esa información al realizar la suscripción (Nafría, en Torres, 2019).

En la evolución de la newsletter, observamos que ha pasado de ser un producto característico del marketing digital a cumplir un relevante papel periodístico (Van der Wel, 2019). También ha sido definida como "la nueva red social" (Isaac, 2019), porque entre otras virtudes, evita al lector algunos inconvenientes de las redes sociales, como la presentación de las noticias mediante algoritmos que no se pueden controlar, o la existencia de bulos (Salaverría et al. 2020). A diferencia de ellas, la newsletter ofrece una comunicación de tipo privado entre autor y suscriptor que potencia vínculos más estrechos (Isaac, 2019).

Para los medios, los boletines tienen un efecto positivo en sus estrategias de negocio. Datos recientes indican que son bastante efectivos para retener suscriptores y atraer nuevos usuarios. The Washington Post, por ejemplo, con 70 boletines diferentes, manifiesta que los destinatarios de boletines consumen tres veces más contenido que quienes no lo son (Newman et al. 2019).

$\mathrm{Su}$ función de filtrado de contenidos frente al exceso de "ruido" de la Red sería otro elemento que explicaría su éxito. Charo Marcos, editora de Kloshletter, señala que funcionan porque "los medios empiezan a darse cuenta de que en las redes hay demasiado ruido" (Marcos, en Tones, 2019). Es así como se han convertido en un canal muy propicio para la realización de lo que se conoce como curación de contenidos (Rojas-Torrijos y González-Alba, 2018). La curación de contenidos periodística se puede definir como: "Un complejo de actividades que incluye: 1) búsqueda y monitorización, 2) selección, 3) análisis y verificación, 4) gestión y edición, y 5) caracterización o sense making de informaciones publicadas en la web, con el objetivo de producir o mejorar productos periodísticos, lo que implica la (6) difusión de tales productos a través de plataformas digitales" (Guallar y Codina, 2018).

El concepto de curación de contenidos proviene del marketing digital (Bhargava, 2009) y se ha extendido a diversas disciplinas y profesiones, desde la gestión de información al periodismo o la educación (Guallar y Leiva-Aguilera, 2013). Concretamente la curación en periodismo ha sido tratada por un buen número de investigadores como: Bradshaw, 2013; Thorsen, 2013; Cappelletti-Junior y Domínguez-Quintas, 2014; Bruns y Highfield, 2015; Guerrini, 2015; Jarvis, 2015; Cui y Liu, 2017; Díaz-Arias, 2017; López-Meri y Casero-Ripollés, 2017; Noguera-Vivo, 2017; Guallar, 2017a,b; Bruns, 2018; Guallar y Codina, 2018; Rojas-Torrijos y González-Alba, 2018; Codina y Guallar, 2019.

En este contexto, presentamos un estudio sobre las newsletters de los medios de comunicación, abordando cómo llevan a cabo la curación de contenidos. Este artículo se inscribe así en la línea de investigaciones del área de Comunicación que estudian el uso de la curación por parte de medios periodísticos.

Hay que señalar que en la literatura de Comunicación existe una notable bibliografía sobre curación en medios sociales, que analiza las prácticas de compartir noticias en redes sociales por parte de los ciudadanos y enlaza con las investigaciones acerca de las audiencias activas en periodismo. En esta línea son relevantes los trabajos de Bruns (por ejemplo, Bruns y Highfield, 2015, o Bruns, 2018) y se puede consultar una revisión de este enfoque en Masip et al. 2019. 
Nuestro enfoque difiere de este último, y se sitúa en la línea de trabajos que estudian el uso de la curación por los propios medios de comunicación. En esta línea, investigaciones anteriores han analizado su uso en webs de diarios (Cui y Liu, 2017), artículos (Guallar 2017a), newsletters (RojasTorrijos y González-Alba, 2018) o plataformas ya desaparecidas como Storify (Cappelletti-Junior y Dominguez-Quintas, 2014). En el precedente más directo de nuestro trabajo, Rojas-Torrijos y González-Alba (2018), analizan los boletines de tres diarios (El país, El español y El independiente), sus contenidos y su modelo de negocio. Se trata de un trabajo exploratorio y descriptivo, a diferencia del nuestro que tiene una intencionalidad evaluativa, como se detallará a continuación.

\section{Objetivos}

Nuestra finalidad es analizar y evaluar el uso de la curación de contenidos en las newsletters de la prensa española. Para ello se establecen los siguientes objetivos específicos:

O1. Presentar una radiografía de las características principales de la curación de contenidos en las newsletters de la prensa española.

O2. Establecer cómo es el boletín tipo de curación periodística en España.

O3. Identificar las mejores newsletters de la prensa española en el uso de la curación de noticias y que puedan servir de referente.

O4. Establecer una clasificación de los medios periodísticos españoles en el uso de la curación de noticias en newsletters y que puedan servir de referente.

\section{Metodología}

Para conseguir dichos objetivos, se realiza una investigación de metodología evaluativa, que incluye técnicas de análisis experto y análisis de contenido (Glaser y Strauss, 1967; Creswell, 2009; FerranFerrer et al. 2017; Morales-Vargas et al. 2020). La metodología que se emplea en este artículo parte de una línea de investigación consolidada a partir de diversos trabajos de Codina y colaboradores aplicada a medios de comunicación (Codina, 2000; Rodríguez-Martínez et al. 2010, 2012; Guallar et al. 2012; Linares et al. 2016; Pedraza-Jiménez et al. 2016) y tiene en cuenta fundamentalmente el método expuesto en Guallar et al. (2020). Así, este trabajo sigue la orientación metodológica del análisis experto (Morales-Vargas et al. 2020) para realizar la evaluación de los objetos estudiados en base a en un sistema de análisis compuesto de parámetros e indicadores organizados en torno a dos grandes áreas o dimensiones: Contenido y Curación (Guallar et al. 2020). La tabla 1 muestra los parámetros o aspectos generales estudiados en cada dimensión (columna izquierda) y los indicadores que permiten valorar estos parámetros (columna derecha).

Tabla 1. Sistema de análisis de curación de contenidos en periodismo. Tabla resumen de dimensiones, parámetros e indicadores.

\begin{tabular}{|l|l|}
\hline \multicolumn{2}{|c|}{ A. Contenido } \\
\hline A1. Número de contenidos & Cantidad \\
\hline A2. Rango temporal & $\begin{array}{l}\text { Información retrospectiva o atemporal } \\
\text { Información reciente } \\
\text { Información actual } \\
\text { Información en tiempo real }\end{array}$ \\
\hline A3. Procedencia & $\begin{array}{l}\text { Contenido propio } \\
\text { Contenido externo }\end{array}$ \\
\hline A4. Fuente según tipo de organización & Fuentes oficiales \\
\hline
\end{tabular}


RLCS, Revista Latina de Comunicación Social, 79, 47-64

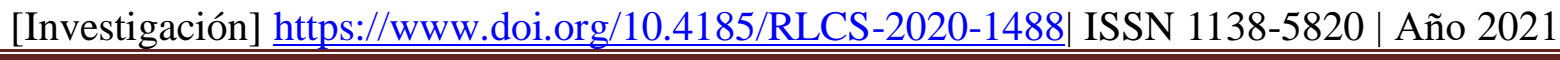

\begin{tabular}{|l|l|}
\hline \multicolumn{1}{|l|}{} & Fuentes corporativas \\
& Medios de comunicación \\
& Ciudadanos \\
\hline A5. Fuente según morfología & Sitios web \\
& Blogs \\
& Redes sociales \\
& Fuentes secundarias \\
\hline \multicolumn{1}{|c|}{ B. Curación } & Autoría \\
\hline B1. Autoría & Resumir \\
\hline B2. Técnica de sense making & Comentar \\
& Citar \\
\hline B3. Función del enlace & Storyboarding \\
& Sin modificar \\
& Describir \\
& Contextualizar \\
& Interpretar \\
& Citar fuente \\
& Citar autor \\
& Llamar a la acción \\
\hline
\end{tabular}

Fuente: Elaboración propia

El sistema de puntuación de indicadores es binario (0-1), evaluando la presencia o ausencia de una característica. Solo en un caso, para el indicador Cantidad del parámetro Número de contenidos, la puntuación es múltiple (0-3), con el fin de valorar esta característica con la escala: mal (0); regular (1); bien (2); muy bien (3), (Guallar et al. 2020).

Para la muestra de análisis se ha buscado que fuera lo más amplia posible para captar una mayor representatividad del periodismo digital realizado en España y se han incluido siete diarios tradicionales o legacy media, y siete diarios nativos digitales, denominación que emplearemos para referirnos a aquellos que son exclusivamente digitales, también conocidos en la literatura con otros nombres, como nuevos medios o metamedios (Cabrera Méndez et al. 2019). Asimismo, se ha considerado conveniente incluir medios que son únicamente newsletters, como representativos de una tendencia reciente iniciada en EEUU con publicaciones como The Skimm y que en España tiene una presencia incipiente. Para establecer esta muestra, se consideraron en primer lugar los diarios digitales españoles de más audiencia según la clasificación mensual de los 10 primeros de ComScore entre enero y mayo de 2019, ranking que no experimentó cambios en la composición de la lista en ese período (aunque sí en sus posiciones) (DIR Confidencial, 2019; PR Noticias, 2019). La lista se completó hasta 14 con los datos de OJD Interactiva para medios digitales en abril de 2019 (OJD Interactiva, 2019). Así, se seleccionaron como legacy media: ABC, El Mundo, El País, El Periódico, La Razón, La Vanguardia y 20 Minutos. Como nativos digitales se escogieron: El Confidencial, El Diario.es, El Español, El Nacional, Nació Digital, OK Diario y Público. A ellos se añadieron dos medios que son únicamente boletines, Kloshletter y Mixxio, como representativos en España de este tipo de marca periodística.

De todos ellos se analizaron sus boletines durante dos meses completos, mayo y junio de 2019. Para los boletines diarios, se analizó un día a la semana, el jueves. Para el resto de periodicidades (semanal o mensual), todos los boletines. En cuanto a puntuaciones, se realizó una evaluación individual de cada newsletter puntuando los distintos indicadores establecidos mediante 0-1 puntos; y en un caso, como se ha indicado, 0-3. La puntuación final de cada indicador se obtiene haciendo la media de las puntuaciones obtenidas durante el periodo de análisis. 


\section{Resultados}

\subsection{Características generales}

Se han analizado 84 boletines (el conjunto de datos del estudio está disponible en https://figshare.com/s/0882e0913aafcca8a253) El número de newsletters de cada cabecera es muy diverso: El País cuenta con 31 boletines; La Vanguardia, 9; El Diario.es, 6; 20 Minutos, ABC y La Razón, 5; El Confidencial y El Español, 4; El Mundo, El Periódico y Nació Digital, 3; Público, 2, y El Nacional, Kloshletter, Mixxio y OK Diario un boletín.

Por tipos de medios, son más numerosas las newsletters de medios tradicionales $(72,6 \%)$ que de nativos digitales $(27,4 \%)$, siendo evidente el peso en el conjunto de El país con 31 (36,9\%). Hay también más especializadas $(67,9 \%)$ que generalistas $(32,1 \%)$, y en la periodicidad se aprecia una importante variedad: son mayoritarias las semanales $(37,44 \%)$, si bien las diversas fórmulas de periodicidad diaria o casi diaria (de 4 a 6 días a la semana) hacen un total ligeramente superior del 45,2\% en sus cuatro variantes (lunes a domingo, 26,2\%; lunes a viernes, 16,6\%; y lunes-jueves, lunes-sábado, una cada una, $1,1 \%)$; además, encontramos boletines quincenales $(5,9 \%)$, mensuales $(1,1 \%)$ у рор-ир о temporales $(3,5 \%)$. Las gratuitas en las que se pide el registro al usuario son mayoría (90\%), y únicamente el 9,5\% tienen acceso de pago para suscriptores. Por último, en cuanto a la autoría, 12 de ellas (el 14,2\%) tienen un editor claramente identificado, siendo por tanto más frecuentes $(85,7 \%)$ las identificadas exclusivamente con la cabecera del medio.

\subsection{Análisis de la dimensión Contenidos}

A continuación, se presentan los resultados obtenidos tras el análisis de los parámetros de la dimensión Contenidos. Se ofrece un resumen de resultados y su análisis para cada parámetro, destacando en cada caso los mejores boletines.

\subsubsection{Cantidad de contenidos curados}

Los boletines evaluados con más contenidos curados incluyen en torno a 30 piezas informativas. Tomando como referencia este valor, la valoración del parámetro se realizó siguiendo como sistema de puntuación: 1-10 contenidos curados: 1 punto, 11-20: 2 puntos, $\geq 21: 3$ puntos. Un dato significativo es que casi la mitad de las newsletters analizadas (40, el 47,6\%) se encuentran en la franja baja, al ofrecer menos de 10 contenidos (1 punto). En el otro extremo, siete boletines pertenecientes a cinco cabeceras obtienen la puntuación más alta (3 puntos): El despertador, El español y Mientras dormías (El español), Adelanto para socios (El Diario.es), El Mundo portada (El Mundo), Claves del día (La Razón) y Mixxio. Se pueden añadir a ese grupo, con una puntuación cercana, dos boletines más: Kloshletter y Ocio (La Razón).

\subsubsection{Rango temporal}

Se consideran cuatro posibilidades, siguiendo las taxonomías de Guallar (2017a, 2017b): Información retrospectiva, la publicada en meses o años anteriores; Información reciente, de los últimos días y semanas; Información actual, de las últimas 24 horas; Información en tiempo real, es decir, en directo o en actualización constante. En nuestro análisis, un boletín obtiene un punto por cada tipo de información que cubre, siendo la puntuación máxima alcanzable 4 puntos. Los boletines analizados muestran hasta 3 rangos temporales en los contenidos curados, obteniendo 3 puntos por ello (o puntuaciones próximas a 3). Con un máximo de 4 no se ha identificado ningún caso. Los mejores boletines son: El País escaparate, El País in English, Planeta futuro, Cinco días y Especial elecciones 
(El País); Mixxio; Desalambre, Consumo claro y Cuarto propio (El Diario.es); El despertador y La bruíxola (Nació Digital); y Mientras dormías (El Español). Estos boletines se sitúan a la cabeza de las buenas prácticas en diversidad temporal de los contenidos, destacando 5 boletines de un mismo medio, El País. Se observa también en conjunto que alrededor del 60\% curan dos rangos temporales: o bien en todos los casos ( 26 boletines, 30,9\%) o en ocasiones ( 24 boletines, $28,5 \%$ ), estando siempre presente en estos casos la información actual, de las últimas 24 horas, más un segundo tipo de información. Un último grupo de $18(21,4 \%)$ curan solo un rango temporal, que es, en todos los casos menos en uno, también el de información actual. Por tanto, se constata que este rango temporal es el hegemónico de la curación en newsletters, estando siempre presente en 75 boletines $(89,2 \%)$.

\subsubsection{Procedencia}

Se tiene en cuenta si los contenidos curados han sido elaborados por el propio medio o si son externos al mismo. Los resultados muestran que aproximadamente una quinta parte de boletines (9; el 19,7\%) cumplen la máxima puntuación al combinar siempre contenidos propios y externos: Los imperdibles (El Confidencial), Desalambre (El Diario.es), El despertador y Mientras dormías (El Español), El País escaparate y Formación (El País), Internacional (La Razón), Mixxio y La bruíxola (Nació Digital). Asimismo, otros 5 curan los dos tipos de contenidos, pero no en todas las ocasiones: Cuarto propio (El Diario.es), El despertador (Nació Digital) y La matrioska, Especial elecciones y La newsletter de Kiko Llaneras (El País). Si por una parte es positivo que 9 boletines alcancen la totalidad de la puntuación y que 5 más se aproximen, se constata que una amplísima mayoría (70; el 83,3\%) ofrecen exclusivamente contenido propio. Considerando estos últimos datos, y con la excepción del caso particular de Kloshletter que muestra solamente contenido externo, cabe señalar como un aspecto altamente negativo que 8 medios de comunicación nunca ofrezcan contenidos externos en sus newsletters: ABC, El Mundo, El Nacional, El Periódico, La Vanguardia, OK Diario, Público y 20 Minutos.

\subsubsection{Fuentes de información según tipo de organización}

Se han considerado, a partir de, entre otros, Barnhurst, 2013; Cui y Liu 2017; Guallar y Codina 2018; Orero y Cebrian-Enrique, 2019, cuatro categorías: fuentes oficiales, de organismos de las administraciones públicas; fuentes corporativas, de empresas, asociaciones y otras organizaciones privadas; medios de comunicación; y ciudadanos particulares. Cada boletín recibe un punto por cada tipo de fuente, siendo 4 la puntuación máxima alcanzable. Las mejores posiciones son de boletines con puntuaciones entre 2 y 2,32, y coinciden con varios de los mejor posicionados en el apartado anterior: Ocio (La Razón); Desalambre (El Diario.es); Mixxio; El despertador y Mientras dormías (El Español); El País escaparate y Formación (El País) y La bruíxola (Nació Digital). También destacan en posiciones cercanas El despertador (Nació Digital), La matrioska (El País) y Kloshletter. El análisis global de resultados del parámetro indica que la información curada proviene principalmente de medios de comunicación, siendo así exclusivamente en 70 boletines $(83,3 \%)$, y quedando el pequeño grupo restante de boletines $(17,7 \%)$ como referentes de una curación periodística basada en una mayor diversidad de fuentes.

\subsubsection{Fuentes de información según morfología}

Este apartado tiene en cuenta la morfología de la fuente informativa y complementa al anterior. A partir de las mismas referencias del parámetro anterior, se consideran 4 tipos: sitios webs, blogs, medios sociales y fuentes secundarias, obteniendo los boletines un punto por cada tipo de fuente curada y siendo 4 la puntación máxima. No se ha detectado ningún boletín con contenidos de fuentes secundarias. Las puntuaciones globales han oscilado entre 1 y 2 puntos y los mejores boletines son 
cuatro: El despertador y Mientras dormías (El Español), y Planeta futuro (El País), porque incluyen siempre contenidos de webs y de redes sociales (los dos primeros) o bien de webs y de blogs (en el último caso), así como Mixxio, que destaca por ser el boletín con mayor equilibrio entre las tres tipologías de fuentes. En una posición cercana aparecen Ocio (La Razón), La matrioska (El País), El despertador (Nació Digital) y Kloshletter. En conjunto se constata el predominio de los sitios web como la tipología de fuente informativa más empleada en la curación periodística en newsletters, de manera abrumadora, pues es el único tipo de fuente en 74 boletines $(88,1 \%)$, mientras que en el resto se combina con la presencia complementaria de contenidos de blogs y de redes sociales.

\subsection{Análisis de la dimensión Curación}

\subsubsection{Autoría}

Como se señaló antes, solo 12 newsletters de las 84 analizadas $(14,2 \%)$ cuentan con un editor o responsable, cuya autoría se explicita. En el resto la autoría se traslada al medio de comunicación en conjunto. Al primer grupo se le asigna una puntuación de un punto y al segundo de cero. Los boletines con editor identificado son: Cuarto propio (Ana Requena Aguilar) y The Guardian en español (Javier Biosca Azcoiti), de El Diario.es; El despertador y Mientras dormías (de Paolo Fava, y en el primero de ellas también Pedro J. Ramirez) de El Español; La matrioska (M. Luz Peinado), El País in English (Melissa Kitson) y La newsletter de Kiko Llaneras (Kiko Llaneras), de El País; El despertador (Ferran Casas) y La bruíxola (Joan Serra Carné), de Nació Digital; El Nacional (José Antich); Kloshletter (Charo Marcos) y Mixxio (Alex Barredo). Coinciden en ser newsletters con un nivel apreciable de trabajo editorial de curación, más allá de la selección de noticias.

\subsubsection{Técnicas de sense making}

Se analizan las técnicas para aportar valor a la curación, conocidas con el término de sense making en la literatura (Deshpande, 2013; Guallar y Leiva-Aguilera, 2013; Martínez-Cañadas, 2017). Se adapta la propuesta de Deshpande (2013) al contexto de las newsletters periodísticas, considerando como técnicas: resumir (técnica fundamentalmente informativa); comentar (opinativa o interpretativa); citar (basada en la cita textual al contenido original); y storyboarding (unir mediante narración piezas de formatos diferentes) y asignando a los boletines un punto por cada técnica que utilizan. Los resultados indican que un importante número de boletines (36, el 42,85\%) no emplean ninguna técnica para aportar valor al contenido. Es un dato significativo, y que corresponde a boletines en gran parte automatizados en base a un titular y a veces una entradilla de cada noticia, sin más aportación. El resto de boletines que sí emplean alguna técnica $(48,57,14 \%)$, no suelen emplear una diversidad de ellas, sino que mayoritariamente utilizan una, la de resumir (informativa). Esta es así la empleada de manera generalizada en la curación no automatizada, bien sea en solitario (en 33 casos, 39,28 \%) o bien complementada $(15,17,85 \%)$ con otras técnicas, que son comentar y citar, sin que se haya identificado uso de storyboarding. Las mejores puntuaciones corresponden a 6 boletines: El despertador y Mientras dormías (El Español), El despertador, La bruíxola i Butlletí del judici (Nació Digital) y El Nacional.

\subsubsection{Función del enlace}

Se estudia la función informativa que tiene dentro del boletín cada hipervínculo a un contenido curado, distinguiendo, a partir de Barnhurst (2013), Cui y Liu (2017) y Guallar et al. (2020), las categorías: sin modificar; describir, contextualizar, interpretar, citar fuente, citar autor y llamar a la acción, asignando a los boletines un punto por cada categoría. Los resultados muestran que todas estas funciones de los enlaces se están usando, si bien son mayoría las newsletters que, o bien emplean únicamente la opción de Sin modificar (38; 45,2\%), o bien emplean Sin modificar junto a Describir, 
en todos los casos o en algunos $(35 ; 41,7 \%)$. La utilización de los enlaces es así bastante pobre en casi el $90 \%$ de los casos y solo en un grupo pequeño existe mayor diversidad. Este grupo lo encabezan El despertador y Mientras dormías (El Español), a nivel destacado (4,11 puntos sobre 7), y les siguen otros boletines habituales de las primeras posiciones en otros parámetros: La bruíxola y El despertador (Nació Digital), Desalambre y Cuarto propio (El Diario.es), Especial elecciones, El País in English y La matrioska (El País) y Kloshletter.

\subsection{Análisis por newsletters y medios digitales}

A continuación, se muestra un análisis de los resultados agrupados por boletines y por medios.

\subsubsection{Newsletters}

Los boletines con mejores puntuaciones se muestran en la tabla 2.

Tabla 2. Clasificación de las mejores newsletters.

\begin{tabular}{|l|l|r|}
\hline Newsletter & Medio & Puntuación \\
\hline El despertador & El Español & 19,41 \\
\hline Mientras dormías & El Español & 19,41 \\
\hline La brúixola & Nació Digital & 16,53 \\
\hline Mixxio & Mixxio & 16,13 \\
\hline El despertador & Nació Digital & 15,62 \\
\hline Desalambre & El Diario.es & 14,98 \\
\hline Kloshletter & Kloshletter & 14,84 \\
\hline Cuarto propio & El Diario.es & 13,39 \\
\hline El País escaparate & El País & 13 \\
\hline La matrioska & El País & 13 \\
\hline El País in English & El País & 12,54 \\
\hline Ocio & La Razón & 12,19 \\
\hline The Guardian en español & El Diario.es & 11,84 \\
\hline La newsletter de Kiko Llaneras & El País & 11,25 \\
\hline Planeta futuro & El País & 11 \\
\hline Internacional & La Razón & 10,76 \\
\hline El Nacional & El Nacional & 10,66 \\
\hline Especial elecciones & El País & 10,5 \\
\hline Formación & El País & 10,24 \\
\hline
\end{tabular}

Fuente: Elaboración propia

Dos newsletters de El Español, El despertador y Mientras dormías, obtienen la primera posición de manera clara (19,41 sobre un total ideal de 29). Se pueden considerar por ello las mejores de la prensa española en el período estudiado. También en posiciones cercanas, están La Bruíxola y El despertador (Nació Digital); Desalambre y Cuarto propio (El Diario.es); Mixxio; y Kloshletter. En conjunto, este es el grupo de boletines de referencia en calidad de la curación periodística en España.

En un nivel algo inferior a los anteriores, pero con elementos de calidad apreciables, encontramos siete boletines del diario El País (El País escaparate, El País in English, La matrioska, La newsletter de Kiko 
Llaneras, Planeta futuro, Especial elecciones y Formación), así como The Guardian en español (El Diario.es), Ocio e Internacional (La Razón) y El Nacional.

En los 18 boletines mencionados están presentes diferentes elementos de una curación periodística de calidad y que se han ido detallando en los apartados anteriores: rangos temporales y fuentes de información diversos, contenidos externos junto a los propios, empleo de técnicas de sense making y utilización diversa de los enlaces. Estos elementos son por el contrario prácticamente inexistentes en los boletines situados en las últimas posiciones de nuestro análisis, que son boletines totalmente automatizados y sin aportación de valor añadido.

\subsubsection{Medios digitales}

Se puede establecer una clasificación de medios (tabla 3). Se ha considerado como puntuación final de cada medio la media de la puntuación obtenida por sus diferentes newsletters.

Tabla 3. Clasificación de medios en calidad de sus newsletters.

\begin{tabular}{|l|r|}
\hline Medio & Puntuación \\
\hline Mixx.io & 16,13 \\
\hline Kloshletter & 14,84 \\
\hline El Español & 13,95 \\
\hline Nació Digital & 13,88 \\
\hline El Diario.es & 11,33 \\
\hline El Nacional & 10,66 \\
\hline La Razón & 9,52 \\
\hline El Periódico & 9,50 \\
\hline El País & 8,72 \\
\hline ABC & 7,54 \\
\hline El Confidencial & 7,33 \\
\hline OK diario & 7,33 \\
\hline El Mundo & 7,00 \\
\hline La Vanguardia & 6,89 \\
\hline Público & 6,61 \\
\hline 20 Minutos & 6,08 \\
\hline
\end{tabular}

Fuente: Elaboración propia

En esta clasificación podríamos establecer cuatro zonas.

- Zona superior (16,13 a 13,88 puntos). Ocupan las primeras posiciones Mixxio y Kloshletter, los dos medios en los que el boletín constituye el único producto periodístico (con la matización de que Mixxio también tiene podcast). Junto a ellos, El Español y Nació Digital son los dos diarios digitales españoles que mejor entienden y más apuestan por la curación en newsletters en su oferta informativa. Un dato significativo es que los cuatro son medios exclusivamente digitales, sin ninguna representación en esta zona alta de legacy media.

- Zona intermedia superior (11,3 a 9,5 puntos). Ocupada a partes iguales, por diarios nativos digitales, El Diario.es y El Nacional, y legacy media, La Razón y El Periódico. Son periódicos cuyas newsletters contienen elementos de valor destacables junto a prácticas automatizadas. 
- Zona intermedia inferior $(8,72$ a 7,33). La ocupan también dos medios de cada tipo: El País y ABC; El Confidencial y OK Diario. Son diarios que, aunque presenten algunos elementos de calidad en sus boletines, estos son minoría en el conjunto de su oferta informativa. Esto es particularmente importante en El País: es el medio con la mayor cantidad de boletines, y ello hace que, a pesar de tener algunos de ellos de calidad, en conjunto queda en esta posición.

- Zona inferior (7 a 6,08). Ocupada por tres legacy media (El Mundo, La Vanguardia, 20 Minutos) y uno exclusivamente digital (Público). Estos diarios utilizan los boletines como servicio automatizado de enlaces a contenidos publicados por el propio medio en las horas anteriores y no han dado el paso de ofrecer un producto con más aportación de valor.

\section{Discusión y conclusiones}

En primer lugar, respondiendo al objetivo O1, esta investigación ha permitido realizar una radiografía de las características de la curación periodística en las newsletters de la prensa española. En relación con el contenido curado se puede destacar lo siguiente:

- Son boletines con una cantidad moderada de contenidos, en muchos casos alrededor de 10 por boletín, aunque existe un pequeño grupo que publica mayor cantidad de contenidos, alrededor de 30 por boletín.

- El contenido curado es mayoritariamente de información actual, publicada en las horas anteriores. No obstante, encontramos bastantes casos en los que se combina la información actual con otro rango temporal: información de los últimos días o retrospectiva.

- El contenido curado es casi siempre del propio medio, e incluso 8 diarios de la muestra estudiada nunca ofrecen contenido que no sea de la propia cabecera.

- El contenido curado proviene, mayoritariamente, de cibermedios, siendo escasa la presencia de otras fuentes como medios sociales o webs oficiales.

Así, en cuanto al contenido curado, se ha constatado que la prensa digital sigue privilegiando los enlaces hacia la información propia muy por encima de los enlaces al contenido externo, con lo que se alinea más con aquellas investigaciones que así lo destacan (Fondevila-Gascón y Segura-Jiménez, 2012; Orero y Cebrian-Enrique, 2019), que con otras que señalan una ligera corrección de este fenómeno (Karlsson et al, 2015). Esto tiene implicaciones en la escasa variedad de fuentes de información existente, llegando a sorprender la baja presencia de contenidos de medios sociales, a pesar de que estos se consideran un canal propicio para la curación de noticias (Bruns, 2018; Guallar y Codina, 2018).

Por otra parte, del análisis de cómo se realiza la curación, se puede destacar:

- En cuanto a la autoría, el hecho de que solo el 14\% de las newsletters identifiquen al editor indica una muy baja presencia del concepto de autoría y, en consecuencia, la utilización mayoritaria del boletín como servicio automatizado de noticias de la cabecera.

- En cuanto a técnicas de sense making, un porcentaje de algo más del $40 \%$ no emplea ninguna. De esta forma, se constata, con mayor precisión que con cualquier otro parámetro, el carácter automatizado y sin aportación profesional periodística de un buen número de ellas. Cuando se aplica alguna técnica, la más empleada es la de resumir, siendo por tanto esta la que mejor se adapta al objetivo del boletín periodístico.

- En el tratamiento periodístico de los enlaces, existe en general un uso pobre o limitado de los mismos. En la mayoría de casos, se utiliza el texto original sin modificar o bien con una breve descripción. 
Así, en cuanto a las características de la curación, esta investigación permitiría establecer, a nivel conceptual, dos grandes tipos de curación periodística, que vamos a denominar intelectual y automatizada.

La curación intelectual está realizada por un profesional con autoría identificada y utiliza una o varias técnicas de sense making, con un uso informativo variado de los enlaces.

La curación automatizada no ofrece una aportación periodística ni tiene identificada su autoría, y se basa en una serie de enlaces a los que no se aplica ninguna técnica de sense making ni existe una variedad de uso informativo en los mismos.

Esta conceptualización permite, respecto a la investigación sobre curación, avanzar en las propuestas previas realizadas tanto a nivel general (Deshpande, 2013; Martínez-Cañadas, 2017); como centradas en el periodismo (Guallar, 2017a; Cui y Liu, 2017). Asimismo, permite destacar que la técnica de resumir (summarizing en Deshpande, 2013) y la descripción en el texto del enlace (sourcing curation en Cui y Liu, 2017), constituyen el eje central del estilo de la curación intelectual en newsletters.

En segundo lugar, siguiendo el objetivo O2, podemos definir el boletín periodístico español de la siguiente manera:

El boletín ofrece entre 10 y 20 contenidos curados que han sido publicados en las horas previas en el sitio web del propio medio de comunicación, no tiene firma de editor, no emplea ninguna técnica de sense making, aunque en ocasiones, sí la de resumir, y sus enlaces tienen el mismo texto presente en el contenido original curado o su descripción.

Por tanto, estos boletines presentan unas características más próximas a la curación automatizada que a la intelectual.

En tercer lugar, respondiendo al objetivo O3, el estudio permite ofrecer un ranking de la calidad de la curación en los boletines de la prensa española. Una primera reflexión es que las newsletters de los primeros lugares de la lista no cumplen la caracterización del boletín español tipo que se ha señalado antes. Estos boletines son por el contrario ejemplos de buenas prácticas en curación intelectual, con diferentes elementos de calidad:

- cantidad importante de contenidos curados;

- diversidad de rangos temporales;

- uso de contenidos externos y no únicamente propios;

- diversidad de fuentes de información;

- identificación del editor;

- empleo de más de una técnica de sense making y

- uso periodístico variado de los enlaces.

Este grupo lo integran El despertador y Mientras dormías, (El Español); La bruíxola y El despertador, (Nació Digital); Desalambre y Cuarto propio, (El Diario.es), y los dos medios que son exclusivamente boletines, Mixxio y Kloshletter. Estos se pueden considerar, en consecuencia, como los boletines de referencia en la curación periodística en España.

En cuarto lugar, siguiendo el O4, el análisis por medios de comunicación ofrece constataciones y paradojas respecto al punto anterior. En cuanto a lo primero, los dos medios que son exclusivamente boletines, Mixxio y Kloshletter, junto con las dos cabeceras con los mejores boletines, El Español y Nació Digital, son los primeros de la lista y constituyen una clara referencia para otros medios de 
comunicación digitales. La paradoja principal se produce en el caso de El País, que, a pesar de tener algunos boletines de calidad, en conjunto queda en posición inferior por la cantidad de sus boletines automatizados. Hay que subrayar, asimismo, que en las posiciones inferiores se encuentran cabeceras relevantes en el sistema mediático español, como son El Mundo, La Vanguardia y 20 Minutos, que, sin embargo, no han apostado por la calidad en la curación de sus newsletters.

Por último, cabría señalar algunos logros y limitaciones de este estudio, así como indicaciones para futuras investigaciones. En cuanto a logros se pueden destacar los siguientes: a) es el primer estudio sistemático en la literatura de análisis de la curación en un conjunto representativo de medios de comunicación de un país; b) pone en práctica un sistema de análisis que puede servir de referencia a futuras investigaciones sobre curación; c) establece clasificaciones de newsletters y de medios de comunicación respecto a la calidad de la curación, que pueden orientar futuras actuaciones de los propios medios analizados y de otros; y d) presenta una caracterización de dos tipos de curación periodística, que permite avanzar en su conceptualización.

Entre las limitaciones, al ser el primer estudio sistemático con esta metodología, creemos que se puede y debe refinar en sucesivos estudios posteriores, añadiendo nuevos parámetros o indicadores o modificando los actuales, así como el sistema de puntuación; por ejemplo, en este artículo la puntuación de la mayoría de los indicadores es binaria (0-1), y en algunos se podría optar por una gradación (0-3), para precisar más el análisis.

En lo que se refiere a futuros estudios, se propone trasladar esta metodología a otros conjuntos acotados de productos periodísticos, como newsletters, reportajes multimedia, noticias de la página de inicio, etc., de cibermedios de un país o de varios países. Asimismo, sería interesante profundizar más en las tipologías de fuentes empleadas en la curación de noticias, así como ahondar en la relación entre medios periodísticos y medios sociales desde el punto de vista del papel de estos últimos como fuentes informativas.

\section{Referencias}

Bhargava, R. (2009). Manifesto for the Content Curator: The next big social media job of the tuture? Rohit Bhargava, September 30. https://bit.ly/30rMnR4

Bradshaw, P. (2013). Journalism *is* curation: tips on curation tools and techniques. Online journalism Blog, September 30. https://bit.ly/2Yg4jvq

Bruns, A. (2018). Gatewatching and news curation: Journalism, social media, and the public sphere. New York: Peter Lang.

Bruns, A. y Highfield, T. (2015). From news blogs to news on Twitter: Gatewatching and collaborative news curation. En: S Coleman \& D. Freelon (Eds.), Handbook of digital politics (pp. 325-339). Cheltenham: Edward Elgar Publishing.

Cabrera-Méndez, M., Codina, L. y Salaverría, R. (2019). Qué son y qué no son los nuevos medios. 70 visiones de expertos hispanos. Revista Latina de Comunicación Social, (74), 1506-1520. https://www.doi.org/10.4185/RLCS-2019-1396-79

Cappelletti-Júnior, M. y Domínguez-Quintas, S. (2014). La curaduría de contenidos y la narrativa colaborativa en el Ciberperiodismo: estudio del caso de Storify en el diario digital elpais.com. 
RLCS, Revista Latina de Comunicación Social, 79, 47-64

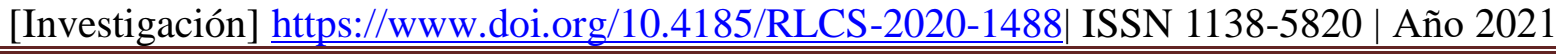

$\begin{array}{lllll}\text { Estudios sobre el mensaje } & \text { periodístico, } & 20(1), & 17-31 .\end{array}$ https://revistas.ucm.es/index.php/ESMP/article/view/45216

Carr, D. (2014). For Email Newsletters, a Death Greatly Exaggerated. The New York Times, June 29. https://nyti.ms/3dRdcCe

Codina, L. (2000). Evaluación de recursos digitales en línea. Revista española de documentación científica, 23(1), 9-44. https://doi.org/10.3989/redc.2000.v23.i1.315

Codina, L. y Guallar, J. (2019). Cura de continguts i fonts d'informació obertes per a comunicadors: relacions estratègiques en un periodisme de qualitat. Comunicació. Revista de recerca i d'anàlisi, 36(2), 71-86. https://doi.org/10.2436/20.3008.01.185

Creswell, J. W. (2009). Research Design. Qualitative, Quantitative and Mixed Methods Approach. Los Ángeles, London: Sage.

Cui, X. y Liu, Y. (2017). How does online news curate linked sources? A content analysis of three online news media. Journalism, 18(7), 852-870. https://doi.org/10.1177/1464884916663621

Deshpande, P. (2013). Six content curation templates for content annotation. The Curata blog, August 13. https://bit.ly/2MIH0EX

Díaz-Arias, R. (2015). Curaduría periodística, una forma de reconstruir el espacio público. Estudios sobre el mensaje periodístico, https://revistas.ucm.es/index.php/ESMP/article/view/51129

DIR Confidencial (2019). El ranking de audiencias de medios digitales, muy cerca de tocar techo tras estrecharse. DIR Confidencial, 23 enero. https://bit.ly/2BIrsie

Ferran-Ferrer, N., Guallar, J., Abadal, E. y Server, A. (2017). Research methods and techniques in Spanish library and information science journals (2012-2014). Information Research, 22(1). http://informationr.net/ir/22-1/paper741.html

Glaser, B. y Strauss, A. L. (1967). The Discovery of Grounded Theory. New Brunswick, London: Aldine.

Guallar, J. (2017a). Artículos de curación de contenidos. Categorías y ejemplos. Anuario ThinkEPI, (11), 210-216. https://doi.org/10.3145/thinkepi.2017.38

Guallar, J. (2017b). Content curation in digital media: Between retrospective and real-time information. En: F. Campos Freire, et al. (Eds.), Media and Metamedia Management, (37-46), Switzerland: Springer International Publishing.

Guallar, J., Abadal, E. y Codina, L. (2012). Sistema de análisis de hemerotecas de prensa digital. Trípodos, (31), 37-64. http://www.tripodos.com/index.php/Facultat_Comunicacio_Blanquerna/article/view/37

Guallar, J. y Codina, L. (2018). Journalistic content curation and news librarianship: Differential characteristics and necessary convergence. El profesional de la información, 27(4), 778-791. https://doi.org/10.3145/epi.2018.jul.07 
Guallar, J. y Leiva-Aguilera, J. (2013). El content curator. Guía básica para el nuevo profesional de internet. Barcelona: Editorial UOC.

Guallar, J., Pedraza-Jiménez, R., Pérez-Montoro, M. y Antón, L. (2020). Curación de contenidos en periodismo. Indicadores y buenas prácticas. Revista española de documentación científica, [en prensa].

Guerrini, F. (2013). Newsroom curators \& independent storytellers: content curation as a new form of journalism. Reuters Institute for the Study of Journalism, University of Oxford. https://bit.ly/3dThpFh

Isaac, M. (2019). The New Social Network That Isn't New at All. The New York Times, March 19. https://nyti.ms/2AsoNcn

Jack, A. (2016). Editorial Email Newsletters. The Medium is Not the Only Message. Reuters Institute for the Study of Journalism. https://bit.ly/2B05g2I

Jarvis, J. (2015). El fin de los medios de comunicación de masas. Barcelona: Gestión 2000.

Laboratorio de Periodismo (2019). Newsletter para periódicos: estrategia y casos de éxito. Laboratoriodeperiodismo.org, 23 mayo. https://bit.ly/2XP76ww

Linares, J., Codina, L., Abadal, E. y Guallar, J. (2016). Periodismo en bases de datos y buscabilidad de la información: protocolo de análisis. Hipertext.net (14), 1-44. https://doi.org/10.2436/20.8050.01.33

López-Meri, A. y Casero-Ripollés, A. (2017). Las estrategias de los periodistas para la construcción de marca personal en Twitter: posicionamiento, curación de contenidos, personalización y especialización. Revista mediterránea de comunicación, 8(1), 59-73. http://dx.doi.org/10.14198/MEDCOM2017.8.1.5

Martínez-Cañadas, E. (2017). Curación de contenidos para bibliotecas. Barcelona: Editorial UOC.

Masip, P., Ruiz-Caballero, C. y Suau, J. (2019). Active audiences and social discussion on the digital public sphere. Review article. El profesional de la información, 28(2), e280204. https://doi.org//10.3145/epi.2019.mar.04

Morales-Vargas, A., Pedraza-Jiménez, R. y Codina, L. (2020). Website quality: An analysis of scientific production. Profesional de la información, 29(5), e290508. https://doi.org/10.3145/epi.2020.sep.08

Newman, N. (2020). The resurgence and importance of email newsletters. En: N. Newman et al., Digital News Report 2020. http://www.digitalnewsreport.org/survey/2020/the-resurgence-andimportance-of-email-newsletters/

Newman, N., Fletcher, R., Kalogeropoulos, A. y Nielsen, R. K. (2019). Digital News Report 2019. Reuters Institute. https://bit.ly/2MKEJcB

Newman, N., Fletcher, R., Schulz, A., Andi, S. y Nielsen, R. K. (2020). Digital News Report 2020. Reuters Institute. https://bit.ly/3drm3JI 
Noguera-Vivo, J. M. (2016). Metamedios y periodismo: revisión panorámica de los nuevos cibermedios. El profesional de la información, 25(3), 341-350. https://doi.org/10.3145/epi.2016.may.03

OJD Interactiva (2019). Medios digitales. https://bit.ly/3fe6d6x

Pedraza-Jiménez, R., Codina, L. y Guallar, J. (2016). Calidad en sitios web: método de análisis general, e-commerce, imágenes, hemerotecas y turismo. Barcelona: Editorial UOC.

Pellicer, M. (2018). Tendencias en el negocio de los newsletters. MiquelPellicer.com, 16 abril https://bit.ly/3fe97rT

PR Noticias (2019). 'La Vanguardia' completa el sorpasso a 'El País' desatando la polémica. $P R$ Noticias, 19 junio. https://bit.ly/3feqEAo

Rodríguez-Martínez, R., Codina, L. y Pedraza-Jiménez, R. (2010). Cibermedios y web 2.0: modelo de análisis y resultado de aplicación. El profesional de la información, 19(1), 35-44. https://doi.org/10.3145/epi.2010.ene.05

Rodríguez-Martínez, R., Codina, L. y Pedraza-Jiménez, R. (2012). Indicadores para la evaluación de la calidad en cibermedios: análisis de la interacción y de la adopción de la Web 2.0. Revista española de documentación científica, 35(1), 61-93 https://doi.org/10.3989/redc.2012.1.858

Rojas-Torrijos, J. L. y González-Alba, J A. (2018). La newsletter como producto periodístico en la búsqueda de nuevos lectores. Estudio de boletines de noticias de El País, El Español y El Independiente. adComunica. Revista Científica de Estrategias, Tendencias e Innovación en Comunicación, (15), 165-195. https://doi.org/10.6035/2174-0992.2018.15.9

Salaverría, R., Buslón, N., López-Pan, F., León, B., López-Goñi, I. y Erviti, M. C. (2020). Desinformación en tiempos de pandemia: tipología de los bulos sobre la Covid-19. El profesional de la información, 29(3), e290315. https://doi.org/10.3145/epi.2020.may.15

Suárez, E. (2020). How to build a successful subscription news business: lessons from Britain and Spain. Reuters Institute Journalist Fellowship paper. https://bit.ly/3feaoPH

Thorsen, E. (2013). Live blogging and social media curation: Challenges and opportunities for journalism. En: K. Fowler-Walt \& S. Allan (Eds.), Journalism: New challenges (123-145), Centre for Journalism \& Communication Research Bournemouth University.

Tones, J. (2019). [Entrevista] Charo Marcos, editora de Kloshletter: «El formato newsletter funciona porque los medios se han dado cuenta de que hay demasiado ruido». Canino, 31 enero. https://bit.ly/3hhrmyx

Torres, A. (2019). Ismael Nafría: «El newsletter es un producto que da alegrías». Sembra Media, 19 septiembre. https://bit.ly/2AeAaVj

Trillo-Domínguez, M. y Alberich-Pascual, J. (2020). Análisis y tipificación de formatos emergentes en el ciberperiodismo español: de la adaptación multimedia a la disrupción transmedia. Estudios sobre el mensaje periodístico, 26(1), 652-668. https://dx.doi.org/10.5209/esmp.67317 
Van der Wel, E. (2019). Newsletters are not a marketing tool, but a journalistic product. Revue, March 28. https://bit.ly/2UxDbXo

\section{AUTORES:}

\section{Javier Guallar}

Universidad de Barcelona. Facultad de Información y Medios Audiovisuales. Centro de investigación en Información, Comunicación y Cultura (CRICC). Doctor por la Universidad de Barcelona, es profesor en la Facultad de Información y Medios Audiovisuales de la Universidad de Barcelona, secretario académico de dicha Facultad y miembro del Centro de investigación en Información, Comunicación y Cultura (CRICC) y del grupo de investigación Cultura y Contenidos Digitales. Sus especialidades de docencia e investigación son la documentación en los medios de comunicación, la curación de contenidos, la gestión de medios sociales y la comunicación académica. Como autor ha publicado tres libros y alrededor de 30 artículos incluidos en WoS o Scopus.

jguallar@ub.edu

Índice H: 20

Orcid ID: https://orcid.org/0000-0002-8601-3990

Google Scholar: https://scholar.google.com/citations?user=L8TSdjsAAAAJ

ResearchGate: https://www.researchgate.net/profile/Javier Guallar

Scopus ID: https://www.scopus.com/authid/detail.uri?authorId=6602474800

\section{Laura Antón}

Graduada en Información y Documentación por la Universidad de Barcelona (UB). Documentalista audiovisual en medios de comunicación de televisión: actualmente en Betevé y anteriormente en TVE y TV3. Sus intereses de investigación se centran en archivos audiovisuales de televisión y curación de contenidos en medios de comunicación.

lanton@beteve.cat

Índice H: 1

Orcid ID: https://orcid.org/0000-0001-8615-5932

Google Scholar: https://scholar.google.com/citations?user=VWDYMmEAAAAJ

ResearchGate: https://www.researchgate.net/profile/Laura Anton4

\section{Rafael Pedraza-Jiménez}

Universidad Pompeu Fabra. Facultad de Comunicación. Doctor por la Universidad de Barcelona, es profesor agregado Serra Húnter del Departamento de Comunicación de la Universidad Pompeu Fabra, y miembro del Grupo de Investigación DigiDoc. Ha participado y/o liderado diferentes proyectos públicos y convenios privados de investigación, relacionados siempre con aspectos de la Web. Imparte docencia en los estudios de Periodismo de la UPF y colabora en másteres de distintas universidades. Como autor ha publicado más de 70 trabajos, más de 20 indexados en WoS o Scopus.

rafael.pedraza@upf.edu

Índice H: 18

Orcid ID: https://orcid.org/0000-0002-6918-6910

Google Scholar: https://scholar.google.com/citations?hl=es\&user=aMM9QaYAAAAJ

ResearchGate: https://www.researchgate.net/search/researcher?q=Rafael+Pedraza-Jim\%C3\%A9nez

Scopus ID: $\underline{\text { https://www.scopus.com/authid/detail.uri?authorId=55890005200 }}$ 


\section{Mario Pérez-Montoro}

Universidad de Barcelona. Facultad de Información y Medios Audiovisuales. Centro de investigación en Información, Comunicación y Cultura (CRICC). Doctor en Filosofía y Ciencias de la Educación por la Universitat de Barcelona, es Catedrático de la Facultad de Información y Medios Audiovisuales de la Universidad de Barcelona y director del Programa de Doctorado en Información y Comunicación en esa Universidad. Su docencia e investigación se centra en el ámbito de la visualización de la información y el diseño de interacción. Ha sido profesor visitante en la Stanford University (EEUU) y la School of Information de la UC Berkeley (EEUU). Tiene más de 30 trabajos indexados en Scopus. perez-montoro@ub.edu

Índice H: 16

Orcid ID: https://orcid.org/0000-0003-2426-8119

Google Scholar: https://scholar.google.es/citations?user=8mMzAYEAAAAJ\&hl=es

ResearchGate: https://www.researchgate.net/profile/Mario_Perez-Montoro

Scopus ID: https://www.scopus.com/authid/detail.uri?authorId=35068527500 